\title{
Image Colorization for Breast Cancer in Magnetic Resonance Imaging
}

\author{
Kok Swee Sim*, Fawaz Sammani \\ Multimedia University, Bukit Beruang, 75450 Melaka, Malaysia. \\ * Corresponding author. Tel.: 06-252-3480; email: kssim@mmu.edu.my \\ Manuscript submitted June 11, 2018; accepted August 18, 2018. \\ doi: 10.17706/ijcee.2018.10.4.265-273
}

\begin{abstract}
This paper is to develop a system for breast cancer detection with colorization to visualize and localize the region of cancerous lesion. With the designed system, radiologist can choose to either run a full scan or partial scan to diagnosis the breasts. This entire system can reduce the time required for analysis and enable more thorough examination for breasts screening. The locations of lesion are colored and exposed out of the MR images for better diagnosis and inspection of the lesion characteristics. The region of interest that is selected allows the system to plot a graph which illustrates transparent details about the lesion. The system in terms of sensitivity and specificity is able to outperform the current manual probing approach.
\end{abstract}

Key words: Image colorization, breast cancer MRI, breast cancer colorization, breast cancer detection.

\section{Introduction}

Breast cancer is the most common cause of death in women and second most common cause of death worldwide. Previously, breast cancer being diagnosis on women from an abnormality appears in mammogram, abnormality on breast skin, a lump or change of breast tissue. The awareness of breast cancer leads to the increasing of women to undergo breast screening such as mammogram for early detection. In Addition, breast cancer mostly can be curable if it is detected early. This helps to reduce the death rate or increase the survival rate of breast cancer carrier. Although breast cancer majority is found on women, males breast cancer does occur but in terms of one percent of all cancer deaths in men. According to an article published by Fritz Frei [1], it states that there is only approximately $1-1.5 \%$ of all breast cancer that occurs in men.

The traditional breast screening method mammogram does provide excellent result for breast cancer detection. However, for some cases such as patients with a dense breast, the screening method is unable to diagnosis the breast because the cancerous lesion hides behind fatty tissues. The low sensitivity ${ }^{1}$ of mammogram encourages researchers to further develop new screening methods for breast cancer detection. One of the newly screening tests is magnetic resonance imaging (MRI), which is used as a supplementary test after mammogram. Several comparisons for mammogram with MRI such as [2] states that the MRI is more efficient and has a higher sensitivity in breast cancer detection compared with traditional breast screening method. The MRI system, as its name implies, generates images by using both

\footnotetext{
1 Sensitivity - The accuracy/percentage of screening test generate positive result.
} 
magnet and radio waves. Images are shown as thin horizontal slices of the breast tissues (depending on the MRI setting), and is able to be observed and studied from different angles. A large number of images are generated and interpreted by a radiologist for each case of breast MRI. Due to the limitation of human eyes, a big number of images need to be analyzed by radiologists. Computer Aided-Detection (CAD) system in MRI is very helpful for radiologists to accurately diagnosis the breast cancer and to overcome this limitation. Comparison of MRI with CAD and traditional MRI [3] shows that MRI with CAD is far more effective and efficient. However, much more false positives are found during the MRI diagnosis with CAD system [4]. Other studies [5]-[7] further compared MRI images with CT images, discussing detection of diseases.

In this study, colorization of breast cancer in MRI is implemented as a CAD system on MRI diagnosis to improve the sensitivity, efficiency and effectiveness of radiologists.

\section{Methodology}

\subsection{Contrast Reagent}

Before contrast agent is injected to the patient's body, the first frame of data acquisition is needed. There are a total of 8 frames which will be taken before and after injection of the contrast reagent. The first frame of the DCE MR images is subtracted from each frame of the images. From the subtracted MR images, if the contrast enhancement is observed in later frames in the lesion with positive mammographic diagnosis, a region of interest is drawn to encompass the enhanced lesion, and a plot of signal intensity-versus-image frame is obtained. With the wash-out [8] of the contrast reagent in the lesion, some classifications are patterned and developed into three different diagrams to identify each lesion case such as benign, suspicious and malignant, as shown in Fig. 1.

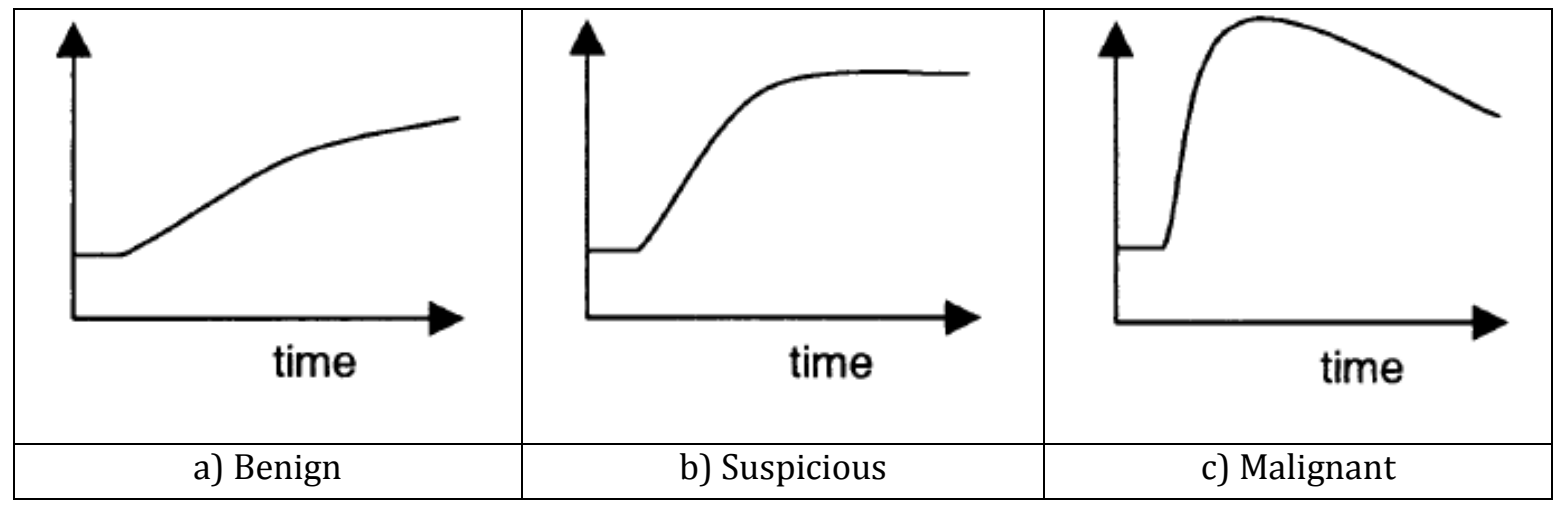

Fig. 1. Patterns of contrast enhancement kinetics [8].

a) Benign lesion - If there is a monotonic increase in signal intensity over six minutes of the examination period. Benign lesion normally lacks of malignancy characteristic and hence it would not grow in size.

b) Suspicious lesion - If the peak signals intensity achieved before three minutes and then maintained for the remainder of the acquisition. Suspicious lesion carries the characteristic and may have the chance to further develop into malignant lesion.

c) Malignant lesion - If there is a decrease in signal intensity immediately after the peak is achieved. The lesion has all the characteristic of cancerous lesion and biopsy is needed for further identification.

\subsection{Data Collection}

A visit to General Hospital (GH) Malacca to obtain and observe the procedures on how MR images are generated was conducted. Besides, the visits also explore how radiologists analyze the MR images with the help of mammography result. The patient's data is basically formed with one pre-contrast image set and 
five post-contrast image set. Each image set contains 144 images (under normal condition) and acquired with the same acquisition technique. The time gap between each image set is approximately one minute. Other MR images such as localizer and subtraction images act as additional resources for further confirmation of the lesion location. Since some of the patient's data provided by GH Malacca does not contains sufficient MR images for this research to analyze, only a few patients' data are selected. However, this research aims to gathers 50 patients with benign lesion, 50 patients with suspicious lesion, and 50 patients for malignant lesion.

\subsection{Region of Interest Scanning}

The selected region of interest inside the MR image is segmented out and analyzed to generate pattern of contrast enhancement graph. Fig. 2a) shows the selection of the region of interest. With the patterned graph, the lesion will be identified among normal tissue, benign lesion, suspicious lesion, and malignant lesion. Fig. 2b) shows the signal intensity graph for the selected region of interest. This ROI function is designed only to scan a set of MR images each time. The image set is selected by referring to the radiologist's result.

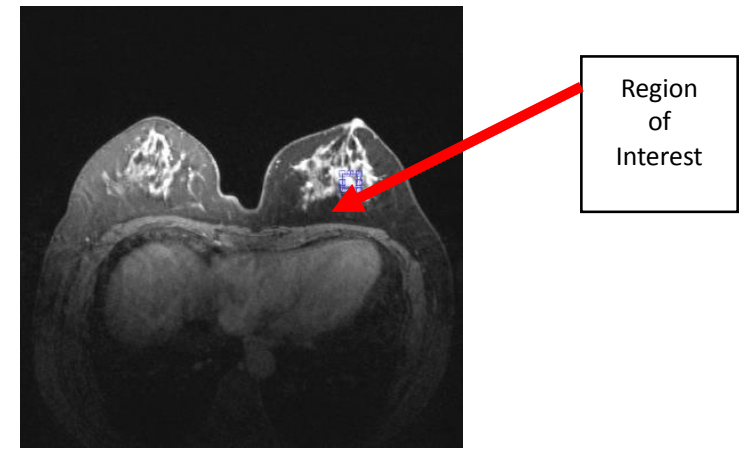

Fig. 2. a) Region of Interest Selection.

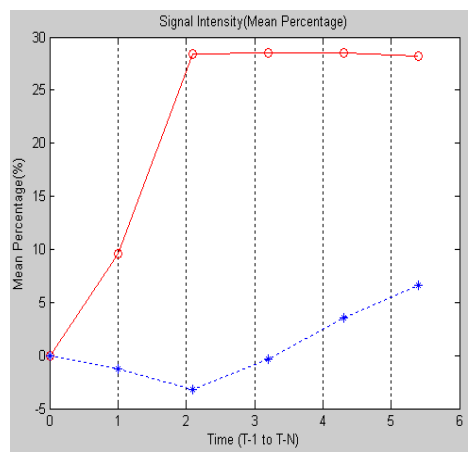

Fig. 2. b) Result of ROI analysis.

\subsection{Full Scanning}

The proposed method of full scanning for the whole breast is to reduce the unnecessary work for radiologist such as randomly probing of suspicious area. The whole process only consumes a small amount of time and it is convenient and effective for radiologist to give better diagnosis. This full scanning system can either work to fully scan set of images or only part of the images which depend on the option that is chosen by user. Fig. 3 is an example of the full scanning view.

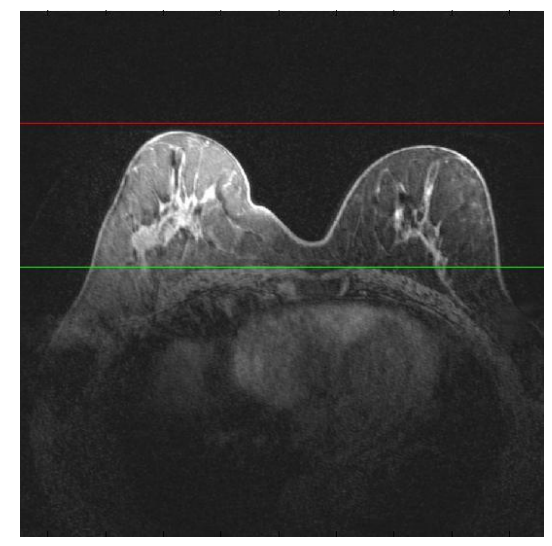

Fig. 3. MR image with selected upper bound (red color) and lower bound (green color). 


\subsection{Lesion Colorization}

Once the lesion(s) is located, the specified color is applied to the image to explore the lesion out from the image. Each color represents difference cases: benign, suspicious or malignant. The color codes are designed by referring to the pattern of contrast enhancement. This technique is similar to the breast thermography where it the digital infrared imaging system is used to generate the colored image. Table 1 shows the color code for each case, benign, suspicious and malignant lesion. Fig. 4 is the representation of the color code that appears on the lesion if the lesion is among benign, suspicious and malignant case. Fig. $5 a$ ) is an example of a lesion found on MR image. The lesion is classified as benign, as shown in Fig. 5b).

Table 1. Color Representation for Each Case

\begin{tabular}{|c|c|}
\hline Case & Color Representation \\
\hline Malignant & red to orange-red \\
\hline Suspicious & light orange-red to light yellow \\
\hline Benign & light green to green \\
\hline
\end{tabular}

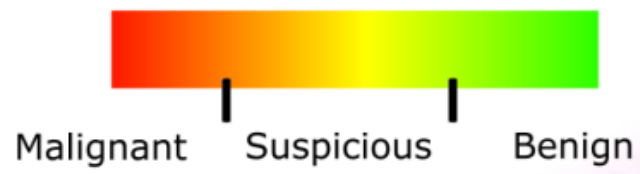

Fig. 4. Lesion color representation.

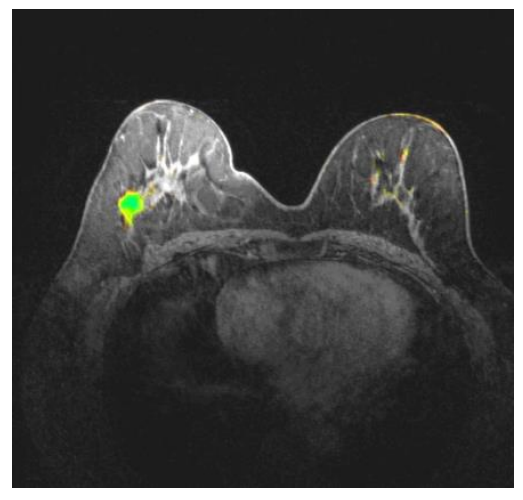

Fig. 5. a) Possible lesion location.

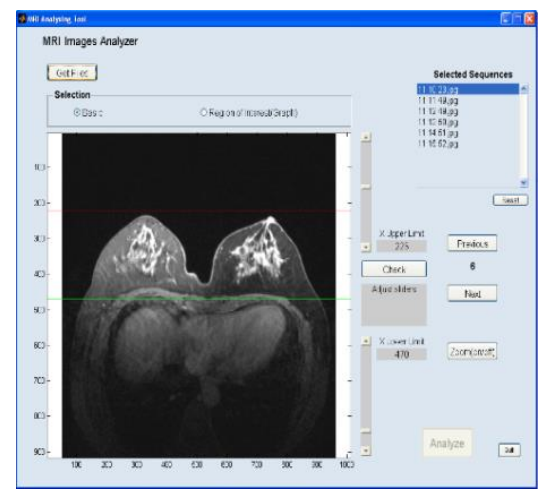

Fig. 5. b) Graphic user interface.

\subsection{Development of Breast Cancer Detection Tool}

After the basic development and analysis for MR image, the last step for this research is to develop a breast cancer detection tool. Similar to any application, a graphic user interface (GUI) is needed for the ease of use. Intelligent detection system is implemented onto this detection tool to reduce unnecessary works.

\subsection{Program Flow Chart and Explanation}

Referring to Fig. 6, at the beginning of the program, the user selects any patient's raw data in Digital Imaging and Communications in Medicine (DICOM) format to be loaded into the program. The raw data loaded into program will be arranged into image sequence. By following, the image sequence will be arranged automatically by the program to prepare the analysis. If any image sequence is not correctly arranged, manual adjustment can be done by the user. At the same time, the contrast of the first image sequence is auto-adjusted. The first image sequence is always evaluated as the original images for further 
analysis. The program is user friendly and allows the user to view each slice of image or select different image sequence and maintain the slice degree. There are two options for user to choose: 'Basic' or 'Region of Interest'.

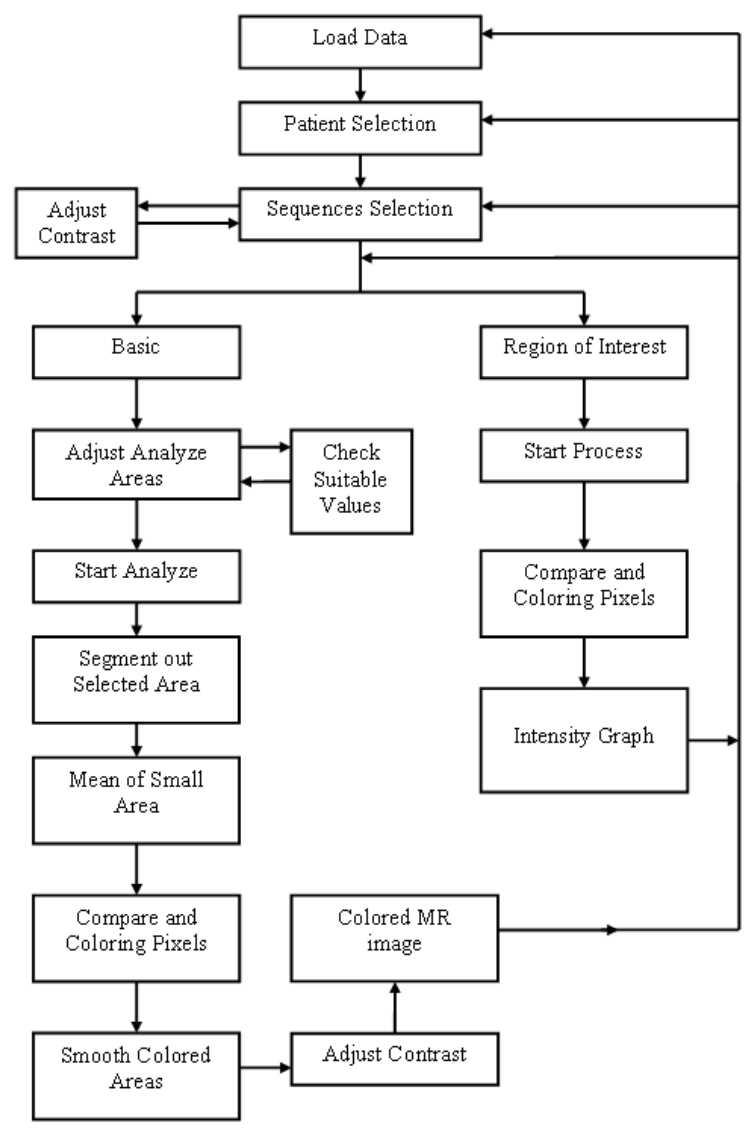

Fig. 6. Program flow chart.

For the first option, the user needs to adjust the upper bound and lower bound of the region of interest. This region of interest is the area which the user wants the program to analyze. While the user adjusts the upper and lower bound, the program will check the suitability of the two bounds. After verifying the suitability, the program will segment out the selected region and then split the images into small blocks.

The program continues the process by calculating the mean value of each small block. For each set of images (slices of different sequences), the program compares the mean value of each small block with same block location in the next sequence of the image. After comparing with all the sequence, the differences between the mean values generate a color code for each small block at the original image (image without contrast adjustment). After the coloring process is completed, the program automatically smoothens the colored image for better view. After this, the contrast of colored image is auto-adjusted and the adjusted image at the program interface is shown. The process will continue until hundred and forty-four image sets are finish analyze by the program.

For second option, the user needs to select two locations that will be analyzed by the program. First the program will detect any mouse input by the user to locate the selected area. Normally, the first location selected will be the lesion area and the second location is normally pointed to the nearest tissues of the first location, which will serve as a reference point. After the selection of two areas, the program will calculate the mean of the selected image and compare it with next image sequence. After the comparison, an 
intensity graph will be plotted by the program to shows the change of the signal intensity of the selected locations. At the same time, the program will draw a square shape for both locations in red (first area) and green (second area) color. The program can repeat using the analysis for other patients without restarting.

\section{Results and Discussion}

Throughout this research, eight patient's data are collected and used for the analysis. Inside each case (benign, suspicious and malignant), the red circle (not shown in the detection system) in the images indicates the lesion or infected areas. The Signal intensity graph is shown besides each MR image to represent the characteristic of each lesion/area. In the graph, the red line represents the location that is analyzed and the green line represents the reference intensity signal for the image over a sequence of time. For each MR image, T1 relaxation time with Fast Low angle Three Dimension in Traverse plane (T1-FL3D-TRA) technique is used to acquire it but with different TR and TE value each.

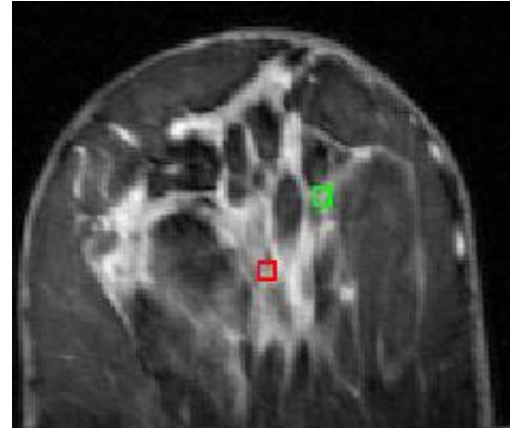

a) Patient 8 (Instance Number 2 63, left breast)

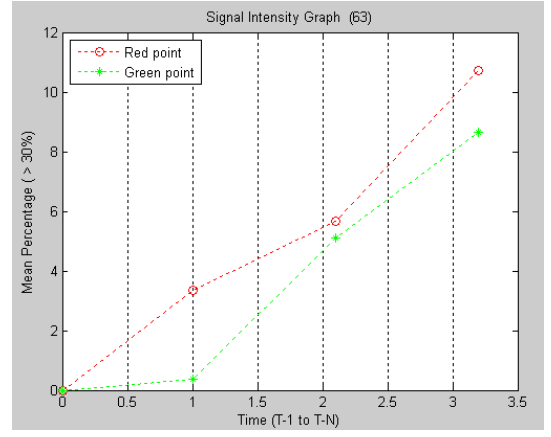

b) Intensity Graph

Fig. 7. Patient 8 location 1.

After the analysis of patient 8's data, the result shows that this patient does not have any risk of breast cancer. To further the analysis, the intensity graph is plotted with the position as shown in Fig. 7b). The result shows a constant rising of signal intensity for both the Red and Green Square, shown in Fig. 7a), which indicates the locations. This situation is caused by the absorption of contrast reagent on breast tissues. However, for normal tissues, the amount of the absorption is normally below $15 \%$ of pre-contrast signal intensity for the same locations.

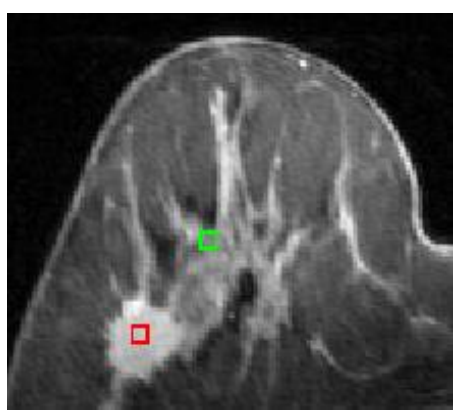

a) Patient 1 (Instance Number 88, left breast)

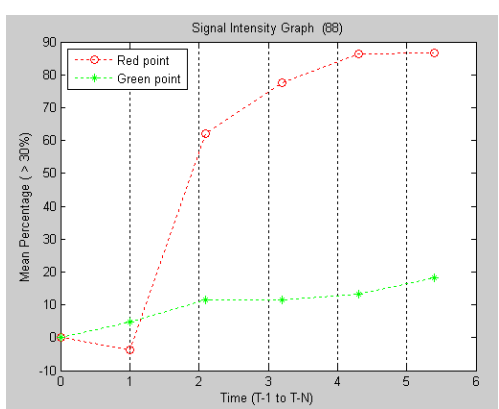

b) Intensity Graph

Fig. 8. Patient 1 lesion 1.

Fig. 8a) shows that patient 1 has a well-defined contour, benign (light green color representation)

2 Instance Number - Structure's field name used by MRI machine to refer the number of images taken in the screening process. 
characteristic lesion. The lesion is located at the middle-left inner side of the patient's left breast. Fig. 8b) shows that the intensity of the lesion area keeps rising within the residence time of contrast reagent. The graph pattern is similar with the "Patterns of Contrast Enhancement Kinetics" benign case as mentioned in the previous section.

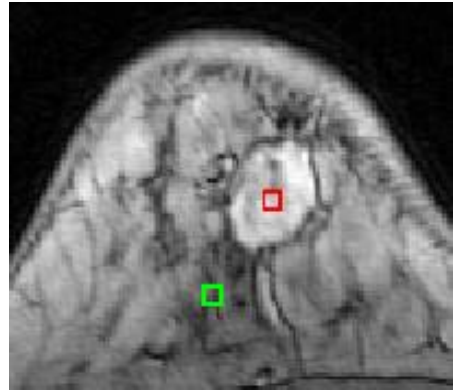

a) Patient 2 (Instance Number 116, left breast)

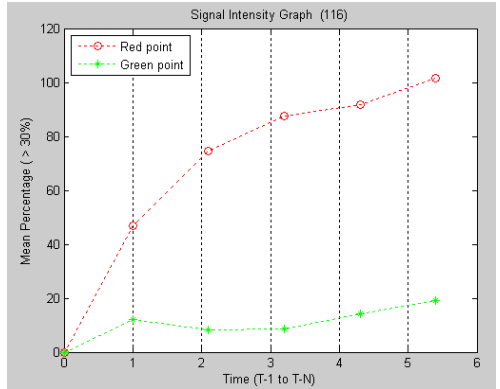

b) Intensity Graph

Fig. 9. Patient 2 lesion 1.

In Fig. 9a), patient 2 has a well-defined contour, benign characteristic lesion locate at middle-right of left breast. Fig. 9b) shows a steady rising graph over the examination period.

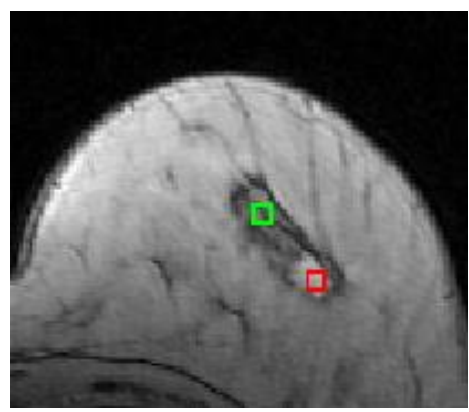

a) Patient 4 (Instance Number 106, right breast)

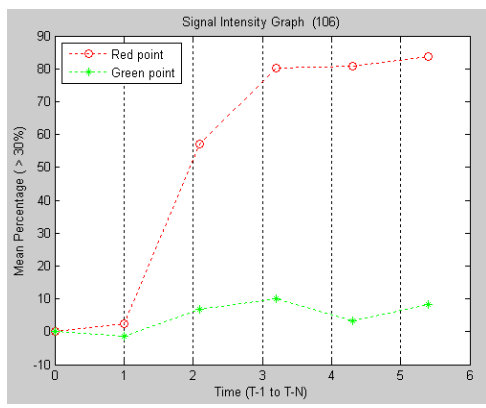

b) Intensity Graph

Fig. 10. Patient 4 lesion 1.

As observed in Fig. 10, the lesion color is with combination of major light green and minor light yellow. With the color representation, this may be classified as benign lesion. From the shape of the lesion, this lesion contains a very well defined contour and no spike available on the contour. Furthermore, the signal intensity of the lesion steadily rises but after three minutes, the absorption rate becomes slower.

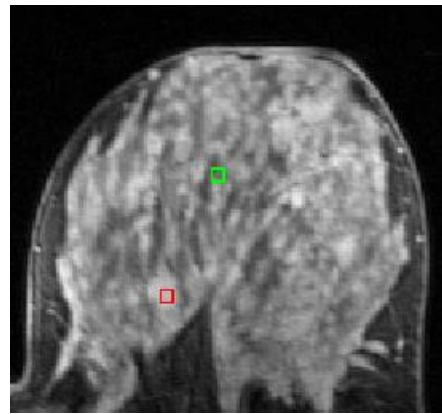

a) Patient 5 (Instance Number 67, right breast)

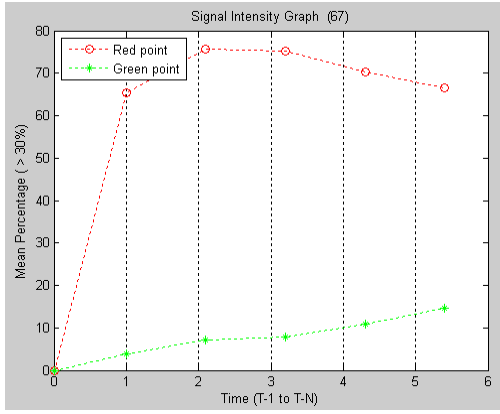

b) Intensity Graph

Fig. 11. Patient 5 lesion 2. 
For patient 5, Fig. 11a) shows that a small lesion is located at the right breast. With the intensity graph plotted at the location as shown in Fig. 11b), it shows that a jump rise at the first minute. After two minutes, a fast decrease rate of intensity occurs at the lesion. This decrement is more than ten percent of the peak value. Hence, this represents malignant type.

Table 2. Detection System and GH Malacca Results

\begin{tabular}{|c|c|c|}
\hline Patient & Detection System Result & Hospital Result \\
\hline 1 & Lesion 1: Benign & Benign \\
\hline 2 & Lesion 1: Benign & \multirow{2}{*}{ Benign } \\
\cline { 2 - 2 } & Lesion 2: Benign & \\
\cline { 2 - 2 } & Lesion 3: Benign & \\
\cline { 2 - 3 } & Lesion 4: Benign & Benign \\
\hline 4 & Lesion 1: Benign & Malignant \\
\hline 5 & Malignant & Normal \\
\hline 8 & Normal & \\
\hline
\end{tabular}

Table 2 shows the comparisons of the detection system results with GH Malacca results. As seen in Table 2 , the final results from the detection system match with the GH Malacca results. Due to bad quality of image sequence, the detection system cannot detect or locate any lesion for patient 10. However, GH Malacca is able to locate the lesion location and plot out a malignant graph pattern. For this case, a false positive analysis result appears in the detection system caused by the slight movement of the patient during the MRI scanning process. This problem needs to be solved so that the quality of the images can be improved and the detection system's sensitivity can be increased.

With these limited raw data of 8 patients, the further testing and improvement on detection system is needed before the actual use as a supplementary/primary test of breast screening.

\section{Acknowledgment}

This research is funded by Fundamental Research Grant Scheme (FRGS), it is a research grant from the Ministry of Higher Education (MOHE), Malaysia. The FRGS project is (Ref: FRGS/1/2016/ICT01/MMU/01/1) 3D Model Reconstruction of Breast Cancer Detection Using Neural Networks.

\section{References}

[1] Fritz, F. Male breast cancer - What you should know about! Retrieved from http://www.cancerhelps.com/knowledge

[2] Fuller, M. S., Lee, C. I., \& Elmore, J. G. (2015). Breast cancer screening: An evidence-based update. The Medical clinics of North America, 99(3), 451-468.

[3] Nikolaou, K., Alkadhi, H., Bamberg, F., Leschka, S., \& Wintersperger, B. J. (2011). MRI and CT in the diagnosis of coronary artery disease: Indications and applications. Insights into Imaging, 2(1), 9-24.

[4] Levman, J. E. D., Gallego-Ortiz, C., Warner, E., et al. (2016). A metric for reducing false positives in the computer-aided detection of breast cancer from dynamic contrast-enhanced magnetic resonance imaging based screening examinations of high-risk women. Journal of Digital Imaging, 29(1), 126-133.

[5] Ashraf, H., Patrick, A., Ernst, W., Christoph, K., Rolf, G., Sebastian, K., Harald, K., Alexander, H., \& Eckart, F. (2011). A prospective study for comparison of MR and CT imaging for detection of coronary artery stenosis. JACC: Cardiovascular Imaging, 4(1), 50-61.

[6] O'Riordan, M. CT more accurate than MRI for ruling out coronary artery disease. Retrieved from 
https://www.medscape.com/viewarticle/716299

[7] Nikolaou, K., Alkadhi, H., Bamberg, F., et al. (2011). MRI and CT in the diagnosis of coronary artery disease: Indications and applications. Insights into Imaging 2(1), 9-24.

[8] Sim K. S., Chia, K. F., Chong, S. S., \& Tso, C. P., \& Fathimah, A. S. (2012). Real Time Based Computer-Aided Design MRI Breast Cancer Detection and Data Management System.

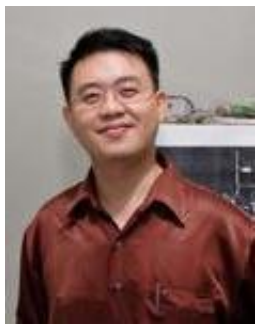

Kok Swee Sim is a professor with Multimedia University, Bukit Beruang, 75450 Melaka, Malaysia. He has won several international awards from the Academic Science Malaysia (ASM) as Top Research Scientists Malaysia (TRSM); Korean innovation and special Awards in 2013, 2014, 2015; the 2005, 2006 and 2011 World Conference in Applied Computing (USA); and 2008 IEEE conference at UK. For national level achievements, he won the Gold Medal Award in the Invention, Innovative \& Technology Exhibition (ITEX) 2008, 2009, 2010, 2013, 2014; Bio Malaysia Award 2009, 2010; Malaysia Technology Expo 2011, AIK2011, AIK2012; Apicta Gold medal award 2014, 2015. He also awarded as MMU best staff in year 2009, 2010 and 2015. In 2016, he was given awards for the TM Kristal Award and two International Championships of World Summit on the Information Society (WSIS) Prizes in the category ICT applications: E-science during the event held in conjunction with WSIS 2016, Geneva, Switzerland. These awards were in the areas of biomedical Engineering (breast cancer detection and brain for early infarct detection). In 2017, he again received another WSIS 2017 International Championship Award on the Automated Pneumatic Glove Sample Stamping Machine for Colorimetric Test. Over all, he has won more than 80 awards which can be found in his website. He is currently working closely with various local and overseas institutions and hospitals such as Department of Electrical and Computer Engineering from National University of Singapore, Centre for Intelligent Systems Research at Deakin University (Australia), Department of Mathematic at Chinese University Hong Kong, Hong Kong University Science and Technology (HKUST), City Hong Kong University (CityU), Perkeso Rehabilitation, Malacca General Hospital, Hospital, Pantai Ayer Keroh and Putra Specialist Hospital.

He has heavily involved in various engineering council namely Institute of Engineer Malaysia (IEM) as Melaka State committee member, secretary, vice chairman, Multimedia University student chapter advisor, Institution of Engineering and Technology (IET) MMU student chapter. He is also the senior member of IEEE, Fellow member of IEM. As professional Engineer, he also registered as Chartered Engineer from IET, Professional Engineer from IEM, International Professional Engineering from IEM and APEC, Asia Pacific Engineer (APEC) from both IEM and APEC, and also Asean Chartered Professional Engineer.

He also serves as fellowship for Malaysia Academic Science Malaysia, senior panel for Engineering Accreditation council and Malaysian Qualifications Agency (MQA) to visit universities to assess university programs. So far, Prof. Sim has filed eight patents and more than 70 copyrights. Prof Sim has brought in National and International funding respectively to support his researches. 